M. Hara

Nagoya Math. J.

Vol. 123 (1991), 171-201

\title{
IDEALS OF BOUNDED HOLOMORPHIC FUNCTIONS ON SIMPLE $n$-SHEETED DISCS
}

\author{
MASARU HARA
}

\section{§ 1. Introduction}

1.1. As usual we denote by $H^{\infty}(R)$ the Banach algebra of bounded holomorphic functions on a Riemann surface $R$ equipped with the supremum norm $\|\cdot\|_{\infty}$. Consider the ideal $I\left(f_{1}, \cdots, f_{m}\right)$ of $H^{\infty}(R)$ generated by functions $f_{1}, \cdots, f_{m}$ in $H^{\infty}(R)$. If a function $g$ in $H^{\infty}(R)$ belongs to $I\left(f_{1}, \cdots, f_{m}\right)$, or equivalently, if there exist $m$ functions $h_{1}, \cdots, h_{m}$ in $H^{\circ}(R)$ with

$$
\sum_{j=1}^{m} f_{j} h_{j}=g
$$

on $R$, then common zero points of $f_{1}, \cdots, f_{m}$ are also zero points of $g$ in the following strong sense:

$$
\sum_{j=1}^{m}\left|f_{j}\right|^{2} \geq \delta|g|^{2}
$$

on $R$ for a positive constant $\delta>0$. The generalized corona problem asks whether the converse is valid or not. In the case $g \equiv 1$ on $R$ the problem is referred to simply as the corona problem.

The simple corona problem was solved by L. Carleson in [C1] in the case where $R$ is the open unit disc $D$ (see, further [K], [G3], [Ga], [BR], [S1], etc). Whether the result can be generalized to arbitrary plane regions is still open, but there are many plane regions for which the problem is in the affirmative (cf. [St1], [B1], [B2], [C2], [GJ], [G1], [Z], [D], $[D W],[N a],[M]$, etc). While there is an example by B. Cole showing that the problem is in the negative for general Riemann surfaces (cf. [G2], [N1]). Also there are many cases in this category where the problem is in the positive (cf. [A1], [A2], [St2], [St3], [Fo], [EM1], [EM2], [H1], [H2], [N2], [JM], etc).

Received January 29, 1991. 
As for the generalized corona problem there is a result by K. Rao [R] that has given a negative answer in the case $R=D$, the open unit disc. We now note the following result (cf. [Ga, p. 329]) which can be reduced to the above mentioned Carleson result by taking $g \equiv 1$ :

The WolfF Theorem. If $f_{1}, \cdots, f_{m}$ and $g$ belong to $H^{\infty}(D)$ with the condition (1.1), then there exist $h_{1}, \cdots, h_{m}$ in $H^{\infty}(D)$ such that

$$
\sum_{j=1}^{m} f_{j} h_{j}=g^{3} .
$$

Thus we must reformulate the generalized corona problem as follows: Determine the smallest positive integer $w(R)$ for any given Riemann surface $R$ such that there exist $h_{1}, \cdots, h_{m}$ in $H^{\infty}(R)$ with

$$
\sum_{j=1}^{m} f_{j} h_{j}=g^{w(R)}
$$

on $R$ where $f_{1}, \cdots, f_{m}$ and $g$ are arbitrary functions in $H^{\infty}(R)$ with (1.1). For convenience we set $w(R)=\infty$ if such an integer can not be found. Therefore the generalized corona theorem asks whether $w(R)<\infty$, and the results of Wolff and Rao imply that

$$
2 \leq w(D) \leq 3 .
$$

It is still not known whether $w(D)=2$ (cf. [Ha], [S2], [Ce]). For certain subalgebras of $H^{\infty}(D)$ the similar considerations are made by [GM] and [Mo]. For general plane regions $R$ it is not known whether $w(R)<\infty$ or not, but there are many plane regions $R$ for which $w(R)<\infty$ and some results concerning the inequality (1.3) have been given (cf. [S2]).

The Cole example mentioned above also shows the existence of general Riemann surface $R$ for which $w(R)=\infty$. In the case of the simple corona problem, we obtained in [HN] the corona theorem with bounds depending only on the sheet number $n$ for $n$-sheeted discs possibly infinitely branched. Our theorem completely generalizes the original Carleson theorem and, in addition, contains earlier works on finite Riemann surfaces. Thus finitely sheeted discs formed an important class for which the simple corona theorem is valid, although it is invalid in general as mentioned earlier. Therefore it is natural and important to study $w(R)$ for finitely sheeted discs $R$ although $w(R)=\infty$ may be the case for general Riemann surfaces $R$.

The main purpose of this paper is to establish the relation 


$$
2 \leq w(R) \leq 4 n-1
$$

for a certain class of $n$-sheeted discs $R$, which we call simple (cf. 1.3 below), possibly infinitely branched and the class trivially contains $D$ so that our result may be viewed as a proper generalization of the above (1.3): Theorems of Wolff and Rao. Some more interpretation of our result will be given in what follows.

1.2. Let $(R, D, \pi)$ be an unbounded $n$-sheeted covering surface of the open unit disc $D=\{|z|<1\}$ with a covering map $\pi$ when $n$ is a positive integer. A Riemann surface $R$ represented as the above $(R, D, \pi)$ is referred to as an $n$-sheeted disc.

We are mainly interested in $w(R)$ for $n$-sheeted discs $R$. We observe the following inequality (cf. Appendix for a proof)

$$
w(R) \geq w(D)
$$

for $n$-sheeted discs $R$.

If an $n$-sheeted disc $R$ has finitely many branch points so that if $R$ is a finite Riemann surface, then we also observe (cf. Appendix for a proof)

$$
w(R)=w(D) .
$$

If $R$ is a covering surface of $D$ of Myrberg type (cf. [SN]), i.e. $H^{\infty}(R)$ $=H^{\infty}(D)$, then (1.6) is of course trivially valid.

1.3. For our purpose to study the dependence of $w(R)$ on the sheet number $n$ for $n$-sheeted discs $R$, we should like to consider $w(n)$ defined by

$$
w(n)=\sup _{R} w(R)
$$

where $R$ runs over the family of all $n$-sheeted discs. For a technical reason we restrict ourselves to the following subfamily of $n$-sheeted discs. An $n$-sheeted disc $R$ is said to be simple or more precisely, simple with respect to a Blaschke product $B(z)$ on $D$, if $R$ is the Riemann surface of an $n$-valued function $\zeta=B(z)^{1 / n}$ for the Blaschke product

$$
B(z)=\prod_{1 \leq \mu \leq N} \frac{-\bar{z}_{\mu}}{\left|z_{\mu}\right|} \frac{z-z_{\mu}}{1-\bar{z}_{\mu} z} \quad(N \leq \infty)
$$

on $D$ whose zeros are all simple where $0 / 0$ is understood to be 1 whenever 
it appears. In addition to $w(n)$ we consider

$$
W(n)=\sup _{R} w(R)
$$

where $R$ runs over the family of all simple $n$-sheeted discs.

We obviously have the inequality $W(n) \leq w(n)$ by definition. For particular cases $n=1$ and 2 we have the equality

$$
W(n)=w(n) \quad(n=1,2) .
$$

In fact, there is only one 1-sheeted disc which coincides with $D$ and is automatically simple. Thus (1.9) is trivial for $n=1$. Actually in this case $W(1)=w(1)=w(D)$. Since any 2-sheeted disc $R$ is either simple or satisfies $H^{\circ}(R)=H^{\infty}(D)$, in the former case we have $w(R) \leq W(2)$ by the definition of $W(2)$, and in the latter case we have $w(R)=w(D) \leq W(2)$ by (1.5). Hence $w(2) \leq W(2)$ which establishes (1.9) for $n=2$.

1.4. As was mentioned at the end of 1.2 a chief object of this paper is to deduce the inequality (1.4) for simple $n$-sheeted discs $R$. The first inequality $w(R) \geq 2$ of (1.4) is a direct consequence of (1.5) :w(R) $\geq w(D)$ with the Rao result $w(D) \geq 2$. Thus the essential part is the proof of the second inequality of $(1.4): w(R) \leq 4 n-1$. Although our proof covers the case $n=1: W(1) \leq 3$, since it is known as the Wolff theorem and we can have better estimate than $w(R) \leq 4 n-1$ for the case $n \geq 2$, we will restrict ourselves only to the case $n \geq 2$. In this case we have the following sharper estimate of $W(n)$ which we assert as the main result of this paper:

The MaIn Theorem. The maximal power $W(n)$ for the generalized corona problem for simple $n$-sheeted discs with $n \geq 2$ satisfies the following inequality

$$
n+1 \leq W(n) \leq 4 n-2 \quad(n \geq 2) .
$$

As was mentioned above our proof for (1.10) works as the proof of

$$
n+1 \leq W(n) \leq 4 n-1 \quad(n \geq 1)
$$

which contains (1.3) as a special case of $n=1$. Here note that $W(1)$ $=w(D)$. We also remark that our proof with a suitable modification works as a proof for $w(R)<\infty$ for $n$-sheeted discs $R$ generated by a Blaschke product whose zeros are not necessarily simple.

The proof of the main theorem will be given in $\S 2$. The proof is 
divided into two parts: the proof of the inequality $W(n) \leq 4 n-2$ and that for $W(n) \geq n+1$. In the proof of $W(n) \leq 4 n-2$ given in the first half of $\S 2$ three results of independent interest stated in 2.2 as Theorems 1,2 and 3 will be made use of. The proofs of Theorems 1,2 and 3 will be given in $\S 3, \S 4$ and $\S 5$, respectively. The proof of $W(n) \geq n+1$ given in the second half of $\S 2$ will also be deduced from Theorem 4 stated in 2.5 which will be proved in $\S 6$. Thus the paper consists of 6 sections.

The last but not the least the author would like to express his sincere thanks to Professor Mitsuru Nakai for his helpful advice and guidance and constant encouragement for many years and also to Dr. Shigeo Segawa for his valuable comments on his work.

\section{§2. Proof of the main theorem}

2.1. Give an $n$-sheeted disc $(R, D, \pi)$ and $f_{1}, \cdots, f_{m}, g$ satisfying (1.1). In order to find solutions of (1.2), we may assume that any of $f_{1}, \cdots, f_{m}, g$ is not identically zero and $\delta=1$. There exists a sequence $\left\{r_{k}\right\}\left(0<r_{k}<1\right)$ converging to 1 such that each set $\pi^{-1}\left(\left\{|z|=r_{k}\right\}\right)$ does not meet the set

$$
\text { \{branch points of } R\} \cup\left(\bigcup_{j=1}^{m} Z\left(f_{j}\right)\right) \cup Z(g)
$$

where $Z(f)$ is the set of zeros of $f$. If we set $D_{k}=\left\{|z|<r_{k}\right\}$ and $R_{k}=$ $\pi^{-1}\left(D_{k}\right)$, then each covering surface $\left(R_{k}, D_{k}, \pi\right)$ is identified with an $n$ sheeted disc $\left(R_{k}^{*}, D, \pi_{k}\right)$. If $R$ is simple, then $R_{k}^{*}$ is simple. We denote by $B_{k}$ a finite Blaschke product which has a simple zero at each point of $\pi_{k}$ (\{branch points of $\left.R_{k}^{*}\right\}$ ). The surface $R_{k}$ is conformally a Riemann surface which the $n$-valued fuuction $\zeta=B_{k}(z)^{1 / n}$ defines. We will give solutions with bounds of $(1.2)$ in $\left(R_{k}, D_{k}, \pi\right)$ so that, by the normal family argument, we will obtain solutions of $(1.2)$ in $(R, D, \pi)$.

2.2. Let $(R, D, \pi)$ be an $n$-sheeted disc. For simplicity a function $f$ on the open unit disc $D$ is identified with the function $f \circ \pi$ on the $n$-sheeted $\operatorname{disc} R$ so that $H^{\infty}(D) \subset H^{\infty}(R)$. For a function $f$ on $R$ we define functions $A(f)$ and $P(f)$ on $D$ by

$$
A(f)(z)=\frac{1}{n} \sum_{k=1}^{n} f\left(p_{k}\right)
$$

and

$$
P(f)(z)=\prod_{k=1}^{n} f\left(p_{k}\right)
$$


for any $z$ in $D$ where $\pi^{-1}(z)=\left\{p_{1}, \cdots, p_{n}\right\}$. In the above sum and product, the multiplicity of the $p_{k}$ is taken into account.

By the reduction in 2.1, in order to prove Main Theorem, we only have to find solutions with bounds of (1.2) for the $n$-sheeted disc $(R, D, \pi)$ and functions $f_{1}, \cdots, f_{m}, g$ in $H^{\infty}(R)$ satisfying (1.1) and having the following two conditions:

(a) $R$ has finitely many branch points;

(b) any of $f_{1}, \cdots, f_{m}, g$ is not identically zero and holomorphic on $\bar{R}$ and does not vanish on $\partial R$.

We denote by $H^{\infty}(\bar{R})$ the class of all functions holomorphic on $\bar{R}$. If in addition $R$ is simple, then there exists a finite Blaschke product $B$ such that the $n$-valued function $\zeta=B(z)^{1 / n}$ defines $R$ and hence $B^{1 / n}$ belongs to $H^{\infty}(R)$. Under this setting we now state three theorems, which are necessary in order to give solutions with bounds of (1.2) in this setting. The first theorem is the following

THEOREM 1. When $R$ is simple with respect to $B$, there exist $h_{1}, \cdots, h_{m}$ in $H^{\circ}(R)$ such that

$$
\sum_{j=1}^{m} f_{j} h_{j}=g^{3} B^{(n-1) / n}
$$

and that

$$
\left\|h_{\jmath}\right\|_{\infty} \leq C \quad(j=1, \cdots, m)
$$

where $C$ is some constant depending only on $\sum_{j=1}^{m}\left\|f_{j}\right\|_{\infty}, m$ and $n$.

The proof of this theorem will be given in $\S 3$.

Theorem 2. Let $R$ be an $n$-sheeted disc. There exist $h_{1}, \cdots, h_{m}$ in $H^{\infty}(R)$ such that

$$
\sum_{j=1}^{m} f_{j} h_{j}=P(g)^{3}
$$

and that

$$
\left\|h_{j}\right\|_{\infty} \leq C
$$

where $C$ is some constant depending only on $\sum_{j=1}^{m}\left\|f_{j}\right\|_{\infty}, m$ and $n$.

In this theorem $R$ need not be simple. The proof will be given in $\S 4$.

THEOREM 3. When $R$ is simple with respect to $B$, there exist $h_{1}, \cdots, h_{n-1}$ in $H^{\infty}(R)$ such that 


$$
g^{n}=\sum_{k=1}^{n-1} h_{k} B^{k / n} g^{n-k}+P(g)
$$

and that

$$
\left\|h_{j}\right\|_{\infty} \leq C
$$

where $C$ is some constant depending only on $\|g\|_{\infty}$ and $n$.

2.3. The inequality $W(n) \leq 4 n-2$ will be derived from those three theorems mentioned in 2.2. We remark that if $h \in H^{\infty}(R)$, then $P(h) / h \in$ $H^{\infty}(R)$ and $\|P(h) / h\|_{\infty} \leq\|h\|_{\infty}^{n-1}$. We denote by $I$ the ideal generated by $f_{1}, \cdots, f_{m}$. Firstly we will show by induction on $k$ that

$$
P(g)^{2} B^{(n-k) / n} g^{n+k-2} \in I \quad(k=1, \cdots, n) .
$$

When $k=1$, from $n \geq 2$, it follows that $2+n+1-2 \geq 3$. Therefore by Theorem 1 , the above for $k=1$ holds. We suppose that the above holds for $1, \cdots, k$. We will show that

$$
P(g)^{2} B^{(n-k-1) / n} g^{n+k-1} \in I .
$$

Replacing $g^{n}$ by the right hand side of (2.5) we see that

$$
\begin{aligned}
P(g)^{2} B^{(n-k-1) / n} g^{n+k-1}=\sum_{\lambda=1}^{n-1} h_{\lambda} P(g)^{2} B^{(n-k-1+\lambda) / n} g^{n-\lambda+k-1} & \\
& +P(g)^{3} B^{(n-k-1) / n} g^{k-1}
\end{aligned}
$$

By the assumption of induction and Theorem 1 each term except the last of the right hand side of the above equality boelngs to $I$ and the last belongs to $I$ by Theorem 2 .

Secondly we will show that

$$
P(g) B^{(n-k) / n} g^{2 n+k-2} \in I \quad(k=1, \cdots, n) .
$$

When $k=1$, we have $1+2 n+1-2 \geq 4$. Therefore by Theorem 1 , the above for $k=1$ holds. We suppose that the above holds for $1, \cdots, k$. We will show that

$$
P(g) B^{(n-k-1) / n} g^{2 n+k-1} \in I .
$$

Replacing $g^{n}$ by the right hand side of (2.5) we see that

$$
\begin{aligned}
P(g) B^{(n-k-1) / n} g^{2 n+k-1}=\sum_{\lambda=1}^{n-1} h_{\lambda} P(g) B^{(n-k-1+\lambda) / n} g^{2 n-\lambda+k-1} & \\
& +P(g)^{2} B^{(n-k-1) / n} g^{n+k-1}
\end{aligned}
$$

By the assumption of induction and Theorem 1 each term except the last 
of the right hand side of the above equality belongs to $I$ and the last belongs to $I$ by the above first result.

Repeating the same argument as in the above two cases, we can show that

$$
B^{(n-k) / n} g^{3 n+k-2} \in I \quad(k=1, \cdots, n) .
$$

Therefore we obtain $g^{4 n-2} \in I$ so that there exist $h_{1}, \cdots, h_{m}$ in $H^{\infty}(R)$ such that

$$
\sum_{j=1}^{m} f_{j} h_{j}=g^{4 n-2}
$$

and

$$
\left\|h_{j}\right\|_{\infty} \leq C
$$

where $C$ is a constant depending on $\sum_{j=1}^{m}\left\|f_{j}\right\|_{\infty}, m$ and $n$.

The proof for $W(n) \leq 4 n-2$ is herewith complete.

2.4. We turn to the proof for $W(n) \geq n+1(n \geq 2)$, which works for $n=1$ as well. For the purpose we prepare the following

Lemma 1. Let $B$ be a Blaschke product and $(R, D, \pi)$ be a simple nsheeted disc which the $n$-valued function $\zeta=B(z)^{1 / n}$ defines. A function $f$ on $R$ is represented uniquely as follows: for any point $p$ in $R$ except for branch points

$$
f(p)=\sum_{k=0}^{n-1} A\left(f B^{k / n}\right)(z) B^{-k / n}(p) \quad(\pi(p)=z) .
$$

Moreover if $f \in H^{\infty}(R)$, then there exist $n$ functions $f_{0}, \cdots, f_{n-1}$ in $H^{\infty}(D)$ uniquely such that

$$
f(p)=\sum_{k=0}^{n-1} f(z) B^{k / n}(p) \quad(\pi(p)=z) .
$$

Proof. Let $\pi^{-1}(z)=\left\{p_{1}, \cdots, p_{n}\right\}$. A linear equation

$$
f\left(p_{i}\right)=\sum_{k=0}^{n-1} x_{k} B^{-k / n}\left(p_{i}\right) \quad(i=1, \cdots, n)
$$

has only one solution by a property of the Vandermonde determinant. Hence there exist functions $s_{0}, \cdots, s_{n-1}$ defined on $D$ uniquely, except for zeros of $B$, such that

$$
f(p)=\sum_{k=0}^{n-1} s_{k}(z) B^{-k / n}(p) \quad(\pi(p)=z) .
$$


Let $\omega=\exp (2 \pi i / n)$. Since

$$
\sum_{k=0}^{n-1} \omega^{i k}=0 \quad(i=1, \cdots, n-1)
$$

we obtain (2.9). When $k \geq 1$, the function $A\left(f B^{k / n}\right)$ vanishes at any zero point of $B$. Hence, if $f$ belongs to $H^{\circ}(R)$, then $A\left(f B^{k / n}\right) / B$ belongs to $H^{\infty}(D)$ so that we obtain (2.10).

2.5. We will show that $W(n) \geq n+1$. For the purpose we take the following theorem which will be proved in $\S 6$.

Theorem 4. There exist Blaschke products $B$ and $G$ satisfying the following three properties:

(a) Both $B$ and $G$ have infinite zero points on the positive real axis;

(b) Any zero of $B$ is simple;

(c) Functions $B$ and $G$ satisfy for any $z$ in $D$

$$
|B(z)| \leq 4\left\{|1-z|+\left|B(z) G(z)^{n}\right|\right\} .
$$

We make use of Blaschke products $G$ and $B$ in the above theorem for the proof of $W(n) \geq n+1$.

Let $R$ be a simple $n$-sheeted disc which $\zeta=B(z)^{1 / n}$ defines. We will show that $W(R) \geq n+1$. Hence $W(n) \geq n+1$. Functions $(1-z)^{1 / n}$ and $G(z)$ belong to $H^{\infty}(D) \subset H^{\infty}(R)$ and satisfy the following inequality:

$$
\left|B^{1 / n}\right|^{2} \leq 4^{2 / n}\left\{\left|(1-z)^{1 / n}\right|^{2}+\left|B^{1 / n} G\right|^{2}\right\} .
$$

Suppose that there exist $f$ and $g$ in $H^{\infty}(R)$ satisfying

$$
(1-z)^{1 / n} f+B^{1 / n} G g=\left(B^{1 / n}\right)^{n} .
$$

By Lemma 1 we have

$$
f=\sum_{k=0}^{n-1} f_{k} B^{k / n} \quad \text { and } \quad g=\sum_{k=0}^{n-1} g_{k} B^{k / n}
$$

where $f_{k}$ and $g_{k}$ belong to $H^{\infty}(D)$. If we replace $f$ and $g$ in (2.13) by the above representations of $f$ and $g$ then, by Lemma 1 , we have the following

$$
(1-z)^{1 / n} f_{0}(z)+G(z) B(z) g_{n-1}(z)=B(z)
$$

for any $z$ in $D$. Since any zero of $B(z)$ is simple, there exists an $h_{0}$ in $H^{\infty}(D)$ such that $f_{0}=h_{0} B$. Hence

$$
(1-z)^{1 / n} h_{0}(z)+G(z) g_{n-1}(z)=1
$$


As $z$ approaches to 1 on the zero sets of $G(z)$ the left hand side of the above converges to zero. This contradiction shows that (2.13) is invalid.

\section{$\S$ 3. Proof of Theorem 1}

3.1. We will prove Theorem 1 . We set

$$
E=\pi\left(\{\text { branch points of } R\} \cup\left(\bigcup_{j=1}^{m} Z\left(f_{j}\right)\right) \cup Z(g)\right) .
$$

Solutions $h_{1}, \cdots, h_{m}$ of (2.1) are constructed as follows. If we set

$$
\psi_{\alpha}=\bar{f}_{\alpha} g\left(\sum_{j=1}^{m}\left|f_{j}\right|^{2}\right)^{-1} \quad(\alpha=1, \cdots, m)
$$

on $R$, then it holds that $\sum_{j=1}^{m} f_{j} \psi_{j}=g$ and $\left|\psi_{\alpha}\right| \leq 1$ on $R$. We set

$$
G_{\alpha \beta}^{k}(z)=A\left(B^{k / n} g \psi_{\alpha} \frac{\partial}{\partial \bar{z}} \psi_{\beta}\right) \quad(\alpha, \beta=1, \cdots, m ; k=0, \cdots, n-1)
$$

on $D \backslash E$ which is infinitely differentiable on $D \backslash E$.

Suppose that we find solutions $b_{\alpha \beta}^{k}$ of the following $\bar{\partial}$-problem

$$
\frac{\partial}{\partial \bar{z}} b_{\alpha \beta}^{k}(z)=G_{\alpha \beta}^{k}(z)
$$

for $z$ in $D \backslash E$. If we set

$$
b_{\alpha \beta}=\sum_{k=0}^{n-1} b_{\alpha \beta}^{k} B^{-k / n}
$$

and

$$
H_{j}=g^{2} \psi_{j}+\sum_{\beta=1}^{m}\left(b_{j \beta}-b_{\beta j}\right) f_{\beta},
$$

then we obtain

$$
\sum_{j=1}^{m} f_{j} H_{j}=g^{3} \quad \text { and } \quad \frac{\partial}{\partial \bar{z}} H_{j}(z)=0
$$

for $z$ in $D \backslash E$. The latter of (3.6) is shown as follows from Lemma 1 and (3.2):

$$
\begin{aligned}
\frac{\partial}{\partial \bar{z}} H_{j} & =g^{2} \frac{\partial}{\partial \bar{z}} \psi_{j}+\sum_{\beta=1}^{m}\left\{\sum_{k=0}^{n-1} G_{j \beta}^{k} B^{-k / n}-\sum_{k=0}^{n-1} G_{\beta j}^{k} B^{-k / n}\right\} f_{\beta} \\
& =g^{2} \frac{\partial}{\partial \bar{z}} \psi_{j}+\sum_{\beta=1}^{m}\left(g \psi_{j} \frac{\partial}{\partial \bar{z}} \psi_{\beta}-g \psi_{\beta} \frac{\partial}{\partial \bar{z}} \psi_{j}\right) f_{\beta} \\
& =g^{2} \frac{\partial}{\partial \bar{z}} \psi_{j}+g \psi_{j} \frac{\partial}{\partial \bar{z}} \sum_{\beta=1}^{m} \psi_{\beta} f_{\beta}-g \sum_{\beta=1}^{m} \psi_{\beta} f_{\beta} \frac{\partial}{\partial \bar{z}} \psi_{j} .
\end{aligned}
$$


If we set

$$
h_{j}=H_{j} B^{(n-1) / n} \quad(j=1, \cdots, m),
$$

then $h_{j}$ will be solutions of Theorem 1 .

3.2. Our next task is to prove (3.3). This will be achieved by proving subsequent five lemmas. We start with the following

LEMma 2. Let $R$ be an $n$-sheeted disc and $f$ be any non-zero function in $H^{\infty}(R)$. Set

$$
E_{f}=\pi\left(\{\text { branch points of } R\} \cup\left(\bigcup_{j=1}^{m} Z\left(f_{j}\right)\right) \cup Z(g) \cup Z(f)\right)
$$

and

$$
G_{\alpha \beta}(z)=A\left(f g \psi_{\alpha} \frac{\partial}{\partial \bar{z}} \psi_{\beta}\right) \quad(\alpha, \beta=1, \cdots, m)
$$

Then the following two inequalities are valid on $D \backslash E_{f}$ :

$$
\left|G_{\alpha \beta}\right|^{2} \leq 4 m \sum_{j=1}^{m} A\left(\left|\frac{d}{d z}\left(f f_{j}\right)\right|^{2}\right)
$$

and

$$
\left|\frac{\partial}{\partial z} G_{\alpha \beta}\right| \leq 10(m+1)\left\{\sum_{j=1}^{m} A\left(\frac{\left|d / d z\left(f f_{j}\right)\right|^{2}}{\left|f f_{j}\right|}\right)+A\left(\frac{|d / d z(f g)|^{2}}{|f g|}\right)\right\} .
$$

Proof. We will prove in the case when $f=1$. If we set $\omega=\sum_{j=1}^{m}\left|f_{j}\right|^{2}$, then we have

$$
\begin{aligned}
\frac{\partial}{\partial \bar{z}} \psi_{\beta}= & g\left\{\frac{\bar{d}}{d z} f_{\beta} \cdot \omega^{-1}-\bar{f}_{\beta} \omega^{-2} \cdot \frac{\partial}{\partial \bar{z}} \omega\right\}, \\
\frac{\partial}{\partial z} \psi_{\beta}= & \bar{f}_{\beta}\left\{\frac{d}{d z} g \cdot \omega^{-1}-g \omega^{-2} \cdot \frac{\partial}{\partial z} \omega\right\}, \\
\frac{\partial^{2}}{\partial z \partial \bar{z}} \psi_{\beta}= & \frac{d}{d z} f_{\beta}\left\{\frac{d}{d z} g \cdot \omega^{-1}-g \omega^{-2} \cdot \frac{\partial}{\partial z} \omega\right\} \\
& -\bar{f}_{\beta}\left\{\frac{d}{d z} g \cdot \omega^{-2} \cdot \frac{\partial}{\partial \bar{z}} \omega-2 g \omega^{-3} \cdot \frac{\partial}{\partial \bar{z}} \omega \cdot \frac{\partial}{\partial z} \omega+g \omega^{-2} \cdot \frac{\partial^{2}}{\partial z \partial \bar{z}} \omega\right\}, \\
\frac{\partial}{\partial z} \omega= & \sum_{j=1}^{m} \frac{d}{d z} f_{j} \cdot \bar{f}_{j}, \quad \frac{\partial}{\partial \bar{z}} \omega=\frac{\frac{\partial}{\partial z} \omega}{z} \text { and } \quad \frac{\partial}{\partial z \partial \bar{z}} \omega=\sum_{j=1}^{m}\left|\frac{d}{d z} f_{j}\right|^{2} \cdot
\end{aligned}
$$

Since $\left|\psi_{j}\right| \leq 1, \omega \geq\left|f_{j}\right|^{2}$ and $\omega \geq|g|^{2}$, we have 


$$
\left|\frac{\partial}{\partial \bar{z}} \psi_{\beta}\right| \leq 2 \sum_{j=1}^{m}\left|\frac{d}{d z} f_{j}\right| \omega^{-1 / 2}
$$

and

$$
\left|\frac{\partial}{\partial z} \psi_{\beta}\right| \leq\left(\left|\frac{d}{d z} g\right|+\sum_{j=1}^{m}\left|\frac{d}{d z} f_{j}\right|\right) \omega^{-1 / 2}
$$

Hence we obtain

$$
\left|g \psi_{\alpha} \frac{\partial}{\partial \bar{z}} \psi_{\cdot \beta}\right| \leq 2 \sum_{j=1}^{m}\left|\frac{d}{d z} f_{j}\right|
$$

By the Schwarz inequality, we obtain (3.8). Similarly we have

$$
\begin{aligned}
\left|\frac{d}{d z} g \cdot \psi_{\alpha} \cdot \frac{\partial}{\partial \bar{z}} \psi_{\beta}\right| & \leq 2\left|\frac{d}{d z} g\right| \omega^{-1 / 2} \sum_{j=1}^{m}\left|\frac{d}{d z} f_{j}\right| \\
& \leq 2\left|\frac{d}{d z} g\right||g|^{-1 / 2} \sum_{j=1}^{m}\left|\frac{d}{d z} f_{j}\right|\left|f_{j}\right|^{-1 / 2} \\
\left|g \frac{\partial}{\partial z} \psi_{\alpha} \cdot \frac{\partial}{\partial \bar{z}} \psi_{\beta}\right| & \leq\left\{2|g|^{1 / 2} \omega^{-1 / 2}\left(\left|\frac{d}{d z} g\right|+\sum_{j=1}^{m}\left|\frac{d}{d z} f_{j}\right|\right)\right\}^{2} \\
& \leq 4\left(\left|\frac{d}{d z} g\right||g|^{-1 / 2}+\sum_{j=1}^{m}\left|\frac{d}{d z} f_{j}\right|\left|f_{j}\right|^{-1 / 2}\right)^{2}
\end{aligned}
$$

and

$$
\begin{aligned}
\left|g \psi_{\alpha} \frac{\partial^{2}}{\partial z \partial \bar{z}} \psi_{\beta}\right| \leq & |g|\left|\frac{d}{d z} f_{\beta}\right|\left|\frac{d}{d z} g\right| \omega^{-1} \\
& +|g|\left(|g|\left|\frac{d}{d z} f_{\beta}\right|+\left|f_{\beta}\right|\left|\frac{d}{d z} g\right|\right)\left(\sum_{j=1}^{m}\left|\frac{d}{d z} f_{j}\right|\left|f_{j}\right|\right) \omega^{-2} \\
& +2\left|f_{\beta}\right||g|^{2}\left(\sum_{j=1}^{m}\left|\frac{d}{d z} f_{j}\right|\left|f_{j}\right|\right)^{2} \omega^{-3}+|g|^{2}\left|f_{\beta}\right|\left(\sum_{j=1}^{m}\left|\frac{d}{d z} f_{j}\right|^{2}\right) \omega^{-2} \\
\leq & 4\left(\left|\frac{d}{d z} g\right||g|^{-1 / 2}+\sum_{j=1}^{m}\left|\frac{d}{d z} f_{j}\right|\left|f_{j}\right|^{-1 / 2}\right)^{2} .
\end{aligned}
$$

Hence we obtain

$$
\begin{aligned}
\left|\frac{\partial}{\partial z}\left(g \psi_{\alpha} \frac{\partial}{\partial \bar{z}} \psi_{\beta}\right)\right| & \leq 10\left(\left|\frac{d}{d z} g\right||g|^{-1 / 2}+\sum_{j=1}^{m}\left|\frac{d}{d z} f_{j}\right|\left|f_{j}\right|^{-1 / 2}\right)^{2} \\
& \leq 10(m+1)\left(\sum_{j=1}^{m}\left|\frac{d}{d z} f_{j}\right|^{2}\left|f_{j}\right|^{-1}+\left|\frac{d}{d z} g\right|^{2}|g|^{-1}\right) .
\end{aligned}
$$

Therefore (3.9) holds.

We next consider the case when $f$ may not be 1 . Observe that $\psi_{\alpha}$ 
is independent of $f$. Based upon this fact the above proof for $f=1$ also works for the general $f$. Namely, we may replace $f_{j}$ and $g$ by $f f_{j}$ and $f g$ in (3.8) and (3.9) established for $f=1$.

3.3. Our second lemma is the following which will be used in the proof of our third lemma: Lemma 4:

Lemma 3. Let $R$ be an n-sheeted disc. For any $f$ in $H^{\infty}(R)$ and any $a$ in $D$, there exists a constant $c$ such that, in some neighborhood of a except for $a$ in $D$,

$$
A\left(\left|\frac{d}{d z} f\right|\right) \leq c|z-a|^{1 / n-1}
$$

and

$$
A\left(\frac{|(d / d z) f|^{2}}{|f|}\right) \leq c|z-a|^{1 / n-2} .
$$

Proof. We may assume that $a=0$. There exists a positive number $\rho$ satisfying the following three properties:

(a) components $\left\{\bar{R}_{i}\right\}$ of $\pi^{-1}(\{|z| \leq \rho\})$ are mutually disjoint;

(b) each component $\bar{R}_{i}$ contains at most one branch point above 0 ;

(c) in each component $\bar{R}_{i}$ the function $f$ does not vanish except for a point above 0 .

The function $f$ in the component $R_{i}$ is represented as follows:

$$
f(z)=\sum_{k \geq 0} c_{k} z^{k / \nu}
$$

where $\nu$ is the sheet number of $R_{i}$. We suppose that $c_{k}=0$ for $1 \leq k \leq K-1$ and $c_{k} \neq 0$. We obtain

$$
\frac{d}{d z} f(z)=\sum_{k \geq K}(k / \nu) c_{k} z^{k / \nu-1}
$$

We consider in the case when $f(0) \neq 0$. For some neighborhood of 0 we have

$$
\left|\frac{d}{d z} f(z)\right| \leq C|z|^{K / \nu-1} \leq C|z|^{1 / n-1}
$$

and

$$
\left|\frac{d}{d z} f(z)\right|^{2}|f(z)|^{-1} \leq C|z|^{2(K / \nu-1)} \leq C|z|^{2 / n-2}
$$


We consider in the case when $f(0)=0$. For some neighborhood of 0 we have

$$
\left|\frac{d}{d z} f(z)\right| \leq C|z|^{K / \nu-1} \leq C|z|^{1 / n-1}
$$

and

$$
\left|\frac{d}{d z} f(z)\right|^{2}|f(z)|^{-1} \leq C|z|^{2(K / \nu-1)-K / \nu} \leq C|z|^{1 / n-2}
$$

Therefore we obtain (3.10) and (3.11).

3.4. Our third lemma is the following which will be used in Lemma 6 below (cf. [Ga, p. 327]).

Lemma 4. Let $R$ be an $n$-sheeted disc. For a non-zero $f$ in $H^{\infty}(\bar{R})$ which does not vanishes on $\partial R$ the measure

$$
A\left(\frac{|(d / d z) f|^{2}}{|f|}\right) \log \frac{1}{|z|} d x d y
$$

is a Carleson measure on $D$ with Carleson norm at most $K\|f\|_{\infty}$, where $K$ is some absolute constant.

Proof. Since

$$
\Delta(|f|)=4 \frac{\partial}{\partial z}\left(\left(\frac{\partial}{\partial \bar{z}} \bar{f}^{1 / 2}\right) f^{1 / 2}\right)=\frac{|(d / d z) f|^{2}}{|f|},
$$

we have

$$
\Delta(A(|f|))=A\left(\frac{|(d / d z) f|^{2}}{|f|}\right)
$$

Let

$$
\begin{aligned}
& \pi(\{\text { branch points of } R\} \cup Z(f)) \cup\{0\}=\left\{a_{0}=0, a_{1}, \cdots, a_{N}\right\}, \\
& \Delta_{j}=\left\{\left|z-a_{j}\right|<\varepsilon\right\}(j=0, \cdots, N) \text { and } D_{\varepsilon}=D \backslash \bigcup_{j=0}^{N} \bar{\Delta}_{j} .
\end{aligned}
$$

For sufficiently small $\varepsilon>0$, it holds that $\bar{\Delta}_{0}, \cdots, \bar{\Delta}_{N}$ are mutually disjoint and each $\bar{\Delta}_{j}$ is contained in $D$. By Green's formula,

$$
\iint_{D_{\varepsilon}} \Delta A(|f|) \log \frac{1}{|z|} d x d y=\int_{\partial D_{\varepsilon}}\left\{\log \frac{1}{|z|} \cdot \frac{\partial}{\partial n} A(|f|)-A(|f|) \frac{\partial}{\partial n} \log \frac{1}{|z|}\right\} d s .
$$

From Lemma 3 and $|\partial / \partial n| f|| \leq|(d / d z) f|$ it follows that 


$$
\Delta A(|f|) \log \frac{1}{|z|} \leq C\left|z-a_{j}\right|^{1 / n-2} \log \frac{1}{|z|}
$$

on $\Delta_{j}$ and

$$
\log \frac{1}{|z|} \cdot\left|\frac{\partial}{\partial n} A(|f|)\right| \leq C\left(\log \frac{1}{|z|}\right)\left|z-a_{j}\right|^{1 / n-1}
$$

on $\partial \Delta_{j}$. By making $\varepsilon \rightarrow 0$, we obtain

$$
\iint_{D} \Delta A(|f|) \log \frac{1}{|z|} d x d y=\int_{\partial D}\{A(|f|)-A(|f|)(0)\} d s .
$$

Hence we have

$$
\iint_{D} A\left(\frac{|(d / d z) f|^{2}}{|f|}\right) \log \frac{1}{|z|} d x d y \leq 2 \pi\|f\|_{\infty} .
$$

Let $S=\left\{r e^{i \theta}: 1-h<r<1,\left|\theta-\theta_{0}\right|<h\right\}$ in $D$. We may assume that $h<1 / 2$. Let

$$
z_{0}=(1-h) e^{i \theta_{0}} \quad \text { and } \quad w=w(z)=\left(z-z_{0}\right) /\left(1-\bar{z}_{0} z\right) .
$$

We set $w=u+i v$. For any $z$ in $S$ we have

$$
\left|1-\bar{z}_{0} z\right|^{2}\left(1-\left|z_{0}\right|^{2}\right)^{-1} \leq c h
$$

and

$$
\begin{aligned}
\log \frac{1}{|z|} \leq 2\left(1-|z|^{2}\right) & =2\left(1-|w|^{2}\right)\left|1-\bar{z}_{0} z\right|^{2}\left(1-\left|z_{0}\right|^{2}\right)^{-1} \\
& \leq 2 \operatorname{ch}\left(1-|w|^{2}\right) \leq(2 \operatorname{ch}) \log \frac{1}{|w|}
\end{aligned}
$$

where $c$ is some absolute constant. By the linear transformation $w=w(z)$, the $n$-sheeted disc $(R, D, \pi)$ is transformed into an $n$-sheeted disc $\left(R_{0}, D, \pi_{0}\right)$. Let

$$
f_{0}(w)=f\left(\left(w+z_{0}\right) /\left(1+\bar{z}_{0} w\right)\right) .
$$

The function $f_{0}$ is holomorphic on $\bar{R}_{0}$ and satisfies $\left\|f_{0}\right\|_{\infty}=\|f\|_{\infty}$. By (3.16), we have

$$
\begin{aligned}
& \iint_{S} A\left(\frac{|(d / d z) f|^{2}}{|f|}\right) \log \frac{1}{|z|} d x d y=\iint_{w(S)} A\left(\frac{\left|(d / d w) f_{0}\right|^{2}}{\left|f_{0}\right|}\right) \log \frac{1}{|z|} d u d v \\
& \quad \leq 2 \operatorname{ch} \iint_{D} A\left(\frac{\left.(\mid d / d w) f_{0}\right|^{2}}{\left|f_{0}\right|}\right) \log \frac{1}{|w|} d u d v \leq 4 \pi \operatorname{ch}\left\|f_{0}\right\|_{\infty} \\
& \quad \leq 4 \pi \operatorname{ch}\|f\|_{\infty} .
\end{aligned}
$$


3.5. The following lemma will be also used in the final Lemma 6 . We denote as usually by $C_{0}^{\infty}(\Omega)$ the class of all infinitely differentiable functions on the region $\Omega$ with compact supports in $\Omega$. Let $\varphi$ satisfy the following properties: $\varphi \in C_{0}^{\infty}(D), \varphi \geq 0$ and $\iint \varphi d x d y 1$. Let $\varphi_{\varepsilon}=\varepsilon^{-2} \varphi\left(\varepsilon^{-1} z\right)$. We denote by $*$ the convolution operation.

Lemma 5. Let $G=G_{\alpha \beta}$ in Lemma 2 and $\rho$ be in $C_{0}^{\infty}(D)$.

(1) The following relation holds:

$$
\frac{\partial}{\partial z}((G \rho) * \varphi)=\left(\frac{\partial}{\partial z}(G \rho)\right) * \varphi
$$

(2) If $\varepsilon$ tends to zero, then the following two integrals converge to zero:

$$
\iint_{D}\left|\left((G \rho) * \varphi_{\varepsilon}\right)^{2}-(G \rho)^{2}\right| \log \frac{1}{|z|} d x d y
$$

and

$$
\iint_{D}\left|\left(\frac{\partial}{\partial z}(G \rho)\right) * \varphi_{\varepsilon}-\frac{\partial}{\partial z}(G \rho)\right| \log \frac{1}{|z|} d x d y .
$$

Proof. By Lemmas 2 and 3 we see that functions $G \rho$ and $\partial / \partial z(G \rho)$ are integrable on C. Hence two functions of (3.17) are infinitely differentiable on $\mathbf{C}$. Give any $\tau$ in $C_{0}^{\infty}(\mathbf{C})$. By virtue of integration by parts and the Fubini theorem we have

$$
\begin{array}{r}
\iint_{\mathrm{C}} \frac{\partial}{\partial z}((G \rho) * \varphi) \tau d x d y=-\iint_{\mathrm{C}}((G \rho) * \varphi) \frac{\partial}{\partial z} \tau d x d y \\
=-\iint_{\mathrm{C}}\left\{\iint_{\mathrm{C}}(G \rho)(z) \frac{\partial}{\partial z} \tau(z+\zeta) d x d y\right\} \varphi(\zeta) d \xi d \eta
\end{array}
$$

We note that $\operatorname{supp}(G \rho(\cdot) \tau(\cdot+\zeta)) \subset D$. We make use of the proof of Lemma 4 for $E_{f}=\left\{a_{0}, \cdots, a_{N}\right\}$. By Green's formula we have

$$
\begin{aligned}
\int_{\partial D_{\varepsilon}}(G \rho)(z) \tau(z+\zeta) d \bar{z}= & \iint_{D_{\varepsilon}} \frac{\partial}{\partial z}(G \rho)(z) \tau(z+\zeta) d z \wedge d \bar{z} \\
& +\iint_{D_{\varepsilon}}(G \rho)(z) \frac{\partial}{\partial z} \tau(z+\zeta) d z \wedge d \bar{z}
\end{aligned}
$$

By Lemmas 2 and 3, if $\varepsilon$ tends to zero, then the left hand side of (3.21) converges to zero. Hence by tracing identities of (3.20) reversely we see 


$$
\iint_{\mathrm{C}} \frac{\partial}{\partial z}((G \rho) * \varphi) \tau d x d y=\iint_{\mathbf{C}}\left(\left(\frac{\partial}{\partial z}(G \rho)\right) * \varphi\right) \tau d x d y
$$

Therefore (1) holds.

Let $\Delta(a ; \delta)=\{z \in \mathbf{C}:|z-a|<\delta\}$. For $\zeta \in \mathbf{C}$, let

$$
\Delta_{1}=\{z \in \Delta(0 ; \delta):|z|<|z-\zeta|\} \text { and } \Delta_{2}=\{z \in \Delta(0 ; \delta):|z|>|z-\zeta|\} .
$$

Since $G \rho$ is square integrable over $\mathbf{C}$ by Lemma 3 , if we fix $\delta$ and make $\varepsilon$ tend to zero, then the integral of (3.18) over $D \backslash \Delta(0 ; \delta)$ converges to zero. We have $|z-\zeta|^{2 / n-2} \leq|z|^{2 / n-2}$ for $z$ in $\Delta_{1} \subset \Delta(0 ; \delta)$. Also we have $|z|^{-1} \leq$ $|z-\zeta|^{-1}$ for $z$ in $\Delta_{2} \subset \Delta(\zeta ; \delta)$. Hence

$$
\iint_{\Delta(0 ; \delta)}|z-\zeta|^{2 / n-2} \log \frac{1}{|z|} d x d y \leq 2 \iint_{\Delta(0 ; \delta)}|z|^{2 / n-2} \log \frac{1}{|z|} d x d y
$$

We will prove that if $D$ is replaced by $\Delta(0 ; \delta)$ in the integral of (3.18), then it can be made arbitrarily small uniformly with respect to $\varepsilon>0$ by making $\delta \rightarrow 0$. We have

$$
\begin{aligned}
\iint_{\Delta(0 ; \delta)}\left|((G \rho) * \varphi)^{2}-(G \rho)^{2}\right| \log \frac{1}{|z|} d x d y \leq & \iint_{\Delta(0 ; \delta)}\left|(G \rho) * \varphi_{\varepsilon}\right|^{2} \log \frac{1}{|z|} d x d y \\
& +\iint_{\Delta(0 ; \delta)}|G \rho|^{2} \log \frac{1}{|z|} d x d y
\end{aligned}
$$

We will evaluate the first term on the right hand side of the above. The second term will be evaluated similarly and actually more easily. By the Schwarz inequailty we have

$$
\begin{aligned}
\iint_{\Delta(0 ; \delta)} & \left\{\iint_{\mathbf{C}}|(G \rho)(z-\zeta)| \varphi_{\varepsilon}(\zeta) d \xi d \eta\right\}^{2} \log \frac{1}{|z|} d x d y \\
\leq & \iint_{\Delta(0 ; \delta)}\left\{\iint_{\mathbf{C}}|(G \rho)(z-\zeta)|^{2} \varphi_{\varepsilon}(\zeta) d \xi d \eta\right\} \log \frac{1}{|z|} d x d y \\
\leq & \iint_{\mathbf{C}}\left\{\iint_{\Delta(0 ; \delta)}|(G \rho)(z-\zeta)|^{2} \log \frac{1}{|z|} d x d y\right\} \varphi_{\varepsilon}(\zeta) d \xi d \eta
\end{aligned}
$$

By using Lemmas 2, 3 and (3.22), consequently, the last term of the above can be made arbitrarily small by making $\delta \rightarrow 0$. This completes the proof for the assertion concerning (3.18). The assertion concerning (3.19) can be proved similarly.

3.6. We are now in the stage to give solutions of the $\ddot{\partial}$-problem (3.5). We state this in Lemma 6. 
Lemma 6. Let $R$ be an $n$-sheeted disc. For a non-zero $f$ in $H^{\infty}(\bar{R})$ which does not vanish on $\partial R$ let $G=G_{\alpha \beta}$ in Lemma 2 .

(1) The measures $|G|^{2} \log (1 /|z|) d x d y$ and $|\partial G / \partial z| \log (1 /|z|) d x d y$ are Craleson measures on $D$ with Carleson norm $C_{1}$.

(2) There exists a function $b$ continuous on $D$ and smooth on $D \backslash E_{f}$ such that

$$
\frac{\partial}{\partial \bar{z}} b=G
$$

on $D \backslash E_{f}$ and that

$$
\varlimsup_{z \rightarrow D D}|b(z)| \leq C_{2} .
$$

Here $C=\max \left(C_{1}, C_{2}\right)$ is some constant depending only on $\sum_{\jmath=1}^{m}\left\|f f_{j}\right\|_{\infty}$ and $m$.

Proof. By Lemmas 2 and 4 we obtain (1). We denote by $c_{i}$ some constant depending only on $\sum_{j=1}^{m}\left\|f f_{j}\right\|_{\infty}$ and $m$. Take a number $0<s<1$ such that $E_{f} \subset\{|z|<s\}$. Let $r_{k}=s+(k / 8)(1-s)(k=1, \cdots, 7)$. We define $\rho$ in $C_{0}^{\infty}([0, \infty))$ such that $0 \leq \rho \leq 1, \rho(t)=1\left(0 \leq t \leq r_{0}\right), \rho(t)=0\left(r_{3} \leq t<\infty\right)$ and $|(d / d t) \rho| \leq 16(1-s)^{-1}$. We set $\rho(z)=\rho(|z|)$ for any $z$ in C. For $\varepsilon<(1 / 8)(1-s)$ we set

$$
G_{\varepsilon}=(G \rho) * \varphi_{\varepsilon}+G(1-\rho),
$$

where $\varphi_{\varepsilon}$ is defined in 3.5. Hence $G_{\varepsilon} \in C^{\infty}(\bar{D})$. We will prove that if $G$ is replaced by $G_{\varepsilon}$ and is sufficiently small, then $G_{\varepsilon}$ satisfies (1). Let

$$
S=\left\{r e^{i \theta}: 1-h \leq r<1,\left|\theta-\theta_{0}\right|<h\right\}
$$

and denote $h$ by $l(S)$. We have

$$
\begin{aligned}
\iint_{S}\left|G_{\varepsilon}\right|^{2} \log \frac{1}{|z|} d x d y \leq & 2 \iint_{S}\left|(G \rho) * \varphi_{\varepsilon}\right|^{2} \log \frac{1}{|z|} d x d y \\
& +2 \iint_{S}|G(1-\rho)|^{2} \log \frac{1}{|z|} d x d y .
\end{aligned}
$$

Since $(G \rho) * \varphi_{\varepsilon}=0$ on $\left\{|z|>r_{5}\right\}$, we may suppose that $l(S)>1-r_{5}$. From Lemma 5 it follows that, for sufficiently small $\varepsilon>0$,

$$
\iint_{D} \|\left.(G \rho) * \varphi_{\varepsilon}\right|^{2}-|G \rho|^{2} \mid \log \frac{1}{|z|} d x d y \leq 1-r_{5} .
$$

Therefore 


$$
\begin{aligned}
\iint_{S}\left|(G \rho) * \varphi_{\varepsilon}\right|^{2} \log \frac{1}{|z|} d x d y & \leq \iint_{S}|G \rho|^{2} \log \frac{1}{|z|} d x d y+1-r_{5} \\
& \leq C_{1} l(S)+l(S) \leq\left(C_{1}+1\right) l(S) .
\end{aligned}
$$

Next we will estimate each term on the right hand side of the following expression:

$$
\frac{\partial}{\partial z} G_{\varepsilon}=\frac{\partial}{\partial z}\left((G \rho) * \varphi_{\varepsilon}\right)+\frac{\partial}{\partial z} G \cdot(1-\rho)-G \frac{\partial}{\partial z} \rho .
$$

From the definition of $\rho$ it follows that

$$
\begin{gathered}
\iint_{S}\left|\frac{\partial}{\partial z} \rho\right|^{2} \log \frac{1}{|z|} d x d y \leq 2 l(S) \int_{0}^{1}\left|\frac{d}{d t} \rho\right|^{2} \log (1 / t) d t \\
\leq 4 \cdot 16^{2}(1-s)^{-2} l(S) \int_{r_{2}}^{r_{3}}(1-t) d t=88 l(S) .
\end{gathered}
$$

By (1) and the Schwarz inequality, we have

$$
\iint_{S}\left|G \frac{\partial}{\partial z} \rho\right| \log \frac{1}{|z|} d x d y \leq c_{1} l(S) .
$$

From Lemma 5 (1), (1) and (3.26) it follows that for sufficiently small $\varepsilon>0$

$$
\iint_{S}\left|\frac{\partial}{\partial z}\left((G \rho) * \varphi_{\varepsilon}\right)\right| \log \frac{1}{|z|} d x d y \leq c_{2} l(S) .
$$

Consequently, if $G$ is replaced by $G_{\varepsilon}$ and $\varepsilon$ is sufficiently small, then two measures of (1) are Carleson measures whose Carleson norms are dominated by $c_{3}$. By Wolffs Theorem ([Ga, p. 322]), there exists a function $b_{\varepsilon}$ continuous on $\bar{D}$ and smooth on $D$ such that

$$
\frac{\partial}{\partial \bar{z}} b_{\varepsilon}=G_{\varepsilon}
$$

on $D$ and

$$
\left\|b_{\varepsilon}\right\|=\sup _{|z|=1}\left|b_{\varepsilon}(z)\right| \leq c_{4}
$$

Let

$$
\hat{G}_{\varepsilon}(z)=\frac{1}{\pi} \iint_{D} \frac{G_{\varepsilon}(\zeta)}{\zeta-z} d \xi d \eta \quad \text { and } \quad \hat{G}(z)=\frac{1}{\pi} \iint_{D} \frac{G(\zeta)}{\zeta-z} d \xi d \eta
$$

From Lemma 3 it follows that $\hat{G}_{\varepsilon}$ and $\hat{G}$ are continuous on $\mathbf{C}$ and $\hat{G}_{\varepsilon}$ converges to $\hat{G}$ uniformly on the boundary $\partial D=\{|z|=1\}$ as $\varepsilon$ tends to 
zero because for any $z$ in $\partial D$ we have

$$
\begin{aligned}
\left|\hat{G}_{\varepsilon}(z)-\hat{G}(z)\right| & \leq \frac{1}{\pi} \iint_{D}\left|(\rho G) * \varphi_{\varepsilon}-\rho G\right| \frac{1}{|\zeta-z|} d \xi d \eta \\
& \leq \frac{8}{\pi}(1-s)^{-1} \iint_{D}\left|(\rho G) * \varphi_{\varepsilon}-\rho G\right| d \xi d \eta .
\end{aligned}
$$

By the generalized Cauchy formula we have the following representation:

$$
b_{\varepsilon}=\hat{G}_{\varepsilon}+h_{\varepsilon}
$$

where $h_{\varepsilon}$ is continuous on $\bar{D}$ and holomorphic on $D$. Take $\varepsilon_{0}>0$ such that $\left|\hat{G}-\hat{G}_{\varepsilon}\right|<1$ on $\partial D$ for $0<\varepsilon \leq \varepsilon_{0}$. Since

$$
h_{\varepsilon}-h_{\varepsilon_{0}}=b_{\varepsilon}-b_{\varepsilon_{0}}-\hat{G}_{\varepsilon}+\hat{G}_{\varepsilon_{0}} \text {, }
$$

we have

$$
\left|h_{\varepsilon}-h_{\varepsilon_{0}}\right| \leq 2 c_{4}+2
$$

on $\partial D$ and hence on $D$ by the maximum principle. Since a set of $\left\{h_{\varepsilon}: \varepsilon_{0} \geq \varepsilon>0\right\}$ is uniformly bounded on $D$, by the normal family argument, some subsequence of $\left\{h_{1 / n}\right\}$ converges to some holomorphic function $h_{0}$ on $D$ uniformly on any compact subset of $D$. If we set

$$
b=\hat{G}+h_{0},
$$

then $b$ is continuous on $D$ and smooth on $D \backslash E$ such that

$$
\frac{\partial}{\partial \bar{z}} b=G
$$

on $D \backslash E$. Furthermore since

$$
b=b_{\varepsilon_{0}}+\hat{G}-\hat{G}_{\varepsilon_{0}}+h_{0}-h_{\varepsilon_{0}},
$$

we have

$$
\varlimsup_{z \rightarrow \partial D}|b(z)| \leq 3 c_{+}+3 .
$$

3.7. Having finished the long preparation we now prove Theorem 1 . By Lemma 6 , there exist functions $b_{\alpha \beta}^{k}(\alpha, \beta=1, \cdots, m ; k=1, \cdots, n-1)$ continuous on $D$ and smooth on $D \backslash E$ such that

$$
\frac{\partial}{\partial \bar{z}} b_{\alpha \beta}^{k}=G_{\alpha \beta}^{k}
$$

on $D \backslash E$ and 


$$
\varlimsup_{z \rightarrow \partial D}\left|b_{\alpha \beta}^{k}(z)\right| \leq C_{1}
$$

where $C_{1}$ is some constant depending only on $\sum_{j=1}^{m}\left\|f_{j}\right\|_{\infty}$ and $m$. If we set

$$
\begin{gathered}
b_{\alpha \beta}=\sum_{k=0}^{n-1} b_{\alpha \beta}^{k} B^{-k / n} \quad(\alpha, \beta=1, \cdots, m), \\
H_{j}=g^{2} \psi_{j}+\sum_{\beta=1}^{m}\left(b_{j \beta}-b_{\beta j}\right) f_{\beta}
\end{gathered}
$$

and

$$
h_{j}=B^{(n-1) / n} H_{j} \quad(j=1, \cdots, m),
$$

then each $h_{j}$ is bounded on $R$, holomorphic on $R \backslash \pi^{-1}(E)$ and satisfies

$$
\varlimsup_{p \rightarrow \partial R}\left|h_{j}(p)\right| \leq C
$$

where $C$ is some constant depending only on $\sum_{j=1}^{m}\left\|f_{j}\right\|_{\infty}, m$ and $n$. An isolated singular point is removable for bounded holomorphic functions. Hence each $h_{j}$ is holomorphic on $R$. By the maximum principle, from (3.34) it follows that $\left\|h_{j}\right\|_{\infty} \leq C$. Since

$$
\sum_{j=1}^{m} f_{j} H_{j}=g^{3},
$$

we have

$$
\sum_{j=1}^{m} f_{j} h_{j}=g^{3} B^{(n-1) / n}
$$

\section{§4. Proof of Theorem 2}

4.1. We will give two lemmas before proving Theorem 2 . Let $\mathbf{C}^{n}$ be the $n$-dimensional complex Cartesian space, $(Z, W)$ the inner product on $\mathbf{C}^{n}$ and $\|Z\|=\sqrt{(Z, Z)}$. Our first lemma is only elementary which will be used in the second essential lemma given later:

Lemma 7. There exists a finite set $\left(Z_{1}, \cdots, Z_{t}\right\}$ in $\mathbf{C}^{n}$ such that $\left\|Z_{i}\right\|$ $=1(i=1, \cdots, t)$ and that

$$
C_{0}=\inf \left\{\sum_{k=1}^{t}\left|\prod_{i=1}^{n}\left(W_{i}, Z_{k}\right)\right|^{2}:\left\|W_{i}\right\|=1(i=1, \cdots, n)\right\}>0 .
$$

Proof. For $W$ in $\mathbf{C}^{n}$, we let

$$
W^{\perp}=\left\{Z \in \mathbf{C}^{n} ;(Z, W)=0\right\} .
$$

For each $\left(W_{1}, \cdots, W_{n}\right)$ which is not the origin of $\left(\mathbf{C}^{n}\right)^{n}$, take a point $Z$ in 
$\mathbf{C}^{n}$ such that $\|Z\|=1$ and $Z$ does not belong to $\bigcup_{j=1}^{n} W_{\frac{1}{j}}$. If we fix $Z$, then there exists a neighborhood $U$ of $\left(W_{1}, \cdots, W_{n}\right)$ such that

$$
\prod_{j=1}^{n}\left(Z, \zeta_{j}\right) \neq 0
$$

for any $\left(\zeta_{1}, \cdots, \zeta_{n}\right)$ in $U$. Since

$$
\left\{\left(W_{1}, \cdots, W_{n}\right) \in\left(\mathbf{C}^{n}\right)^{n} ;\left\|W_{j}\right\|=1(j=1, \cdots, n)\right\}
$$

is compact, we can select a required $\left\{Z_{1}, \cdots, Z_{t}\right\}$.

4.2. We will prove the following lemma which reduces an argument for an $n$-sheeted disc to that for the unit disc.

Lemma 8. For any $F_{1}, \cdots, F_{m}(m \geq n)$ in $H^{\infty}(\bar{R})$, there exist $\theta_{i j}$ in $H^{\infty}(\bar{D})$ and $\tau_{i}$ in $H^{\infty}(\bar{R})(i=1, \cdots, T ; j=1, \cdots, m)$ such that

$$
\left\|\theta_{i j}\right\|_{\infty} \leq C_{1} \quad \text { and }\left\|\tau_{i}\right\|_{\infty} \leq C_{1}(i=1, \cdots, T ; j=1, \cdots, m)
$$

and that

$$
\sum_{j=1}^{m} \sum_{i=0}^{T} \bar{\theta}_{i j} \tau_{i} F_{j}=\sum_{j=1}^{m} \sum_{i=1}^{T}\left|\theta_{i j}\right|^{2} \geq C_{0} P\left(\sum_{j=1}^{m}\left|F_{j}\right|^{2}\right)
$$

where $T$ is a positive integer depending only on $m$ and $n, C_{0}$ a constant depending only on $n$ and $C_{1}$ a constant depending only on $n$ and $\sum_{j=1}^{m}\left\|F_{j}\right\|_{\infty}$.

Proof. Take $Z_{k}=\left(z_{k 1}, \cdots, z_{k n}\right)(k=1, \cdots, t)$ for which Lemma 7 holds. We get the following inequality: for any $W_{1}, \cdots, W_{n}$ in $\mathbf{C}^{n}$

$$
\sum_{k=1}^{t}\left|\prod_{i=1}^{n}\left(W_{i}, Z_{k}\right)\right|^{2} \geq C_{0} \prod_{i=1}^{n}\left\|W_{i}\right\|^{2} .
$$

Let $(R, \pi, D)$ be an $n$-sheeted disc. For any $f$ in $H^{\circ}(\bar{R})$ we have $P(f) \in$ $H^{\infty}(\bar{D})$ and $P(f) \mid f \in H^{\infty}(\bar{R})$. Let $j_{1}, \cdots, j_{n}$ be an increasing sequence defined by terms belonging to $\{1, \cdots, n\}$. We set

$$
\begin{gathered}
J=\left\{j_{1}, \cdots, j_{n}\right\}, \\
\theta_{k J}=P\left(\sum_{i=1}^{n} \overline{z_{k \lambda}} F_{j_{\lambda}}\right) \in H^{\infty}(\bar{D})
\end{gathered}
$$

and

$$
\tau_{k J}=\theta_{k J}\left(\sum_{i=1}^{n} \overline{z_{k \lambda}} F_{j_{\lambda}}\right)^{-1} \in H^{\infty}(\bar{R}) .
$$

If we set 


$$
W_{i}=\left(F_{j_{1}}\left(p_{i}\right), \cdots, F_{j_{n}}\left(p_{i}\right)\right) \in \mathbf{C}^{n},
$$

then we have

$$
\begin{aligned}
\sum_{k=1}^{t}\left|\prod_{i=1}^{n}\left(W_{i}, Z_{k}\right)\right|^{2} & =\sum_{k=1}^{t}\left|\prod_{i=1}^{n}\left(\sum_{\lambda=1}^{n} F_{j_{\lambda}}\left(p_{i}\right) \overline{z_{k \lambda}}\right)\right|^{2} \\
& =\sum_{k=1}^{t}\left|P\left(\sum_{\lambda=1}^{n} \overline{z_{k \lambda}} F_{j_{\lambda}}\right)\right|^{2}=\sum_{k=1}^{t}\left|\theta_{k J}\right|^{2} \\
& =\sum_{k=1}^{t} \sum_{\lambda=1}^{n} \overline{z_{k \lambda} \theta_{k J}} \tau_{k J} F_{j_{\lambda}} .
\end{aligned}
$$

From

$$
\sum_{i=1}^{n}\left\|W_{i}\right\|^{2}=\sum_{i=1}^{n} \sum_{\lambda=1}^{n}\left|F_{j_{\lambda}}\left(p_{i}\right)\right|^{2}=P\left(\sum_{i=1}^{n}\left|F_{j_{\lambda}}\right|^{2}\right)
$$

it follows that

$$
\sum_{\lambda=1}^{n} \sum_{k=1}^{t} \overline{z_{k \lambda} \theta_{k J}} \tau_{k J} F_{j_{\lambda}}=\sum_{k=1}^{t}\left|\theta_{k J}\right|^{2} \geq C_{0} P\left(\sum_{j=1}^{n}\left|F_{j_{\lambda}}\right|^{2}\right) .
$$

Functions $\theta_{i j}$ and $\tau_{i}(i=1, \cdots, T ; j=1, \cdots, m)$ can be defined as follows. There is a positive integer $T$ depending on $m$ and $n$ such that there exists a bijection $\nu$ of

$$
\left\{(k, J): 1 \leq k \leq t, 1 \leq j_{1}<\cdots<j_{n} \leq m\right\}
$$

onto $\{1, \cdots, T\}$. Set

$$
\tau_{i}=\tau_{k J} \text { for } i=\nu(k, J) \text {. }
$$

For any $(i, j)(i=1, \cdots, T ; j=1, \cdots, m)$ there exists a unique $(k, J)$ such that $i=\nu(k, J)$. If the integer $j$ does not belong to $J$, then we set

$$
\theta_{i j}=0 \text {. }
$$

If the integer $j$ belongs to $J$, that is $j=j_{\lambda}$, then we set

$$
\theta_{i j}=z_{k \lambda} \theta_{k J} .
$$

Hence we have

$$
\begin{aligned}
\sum_{j=1}^{m} \sum_{i=1}^{T} \overline{\theta_{i j}} \tau_{i} F_{j} & =\sum_{J} \sum_{k=1}^{t} \sum_{j=1}^{m} \overline{\theta_{\nu(k, J) j}} \tau_{\nu(k, J)} F_{j} \\
& =\sum_{J} \sum_{k=1}^{t} \sum_{j \in J} \overline{\theta_{\nu(k, J) j}} \tau_{\nu(k, J)} F_{j}=\sum_{J} \sum_{k=1}^{t} \sum_{\lambda=1}^{n} \overline{\theta_{\nu(k, r) j_{\lambda}}} \tau_{\nu(k, J)} F_{j_{\lambda}} \\
& =\sum_{J} \sum_{k=1}^{t} \sum_{\lambda=1}^{n} \overline{z_{k \lambda} \theta_{k J}} \tau_{k J} F_{j_{\lambda}} .
\end{aligned}
$$


By the similar argument and $\left\|Z_{k}\right\|=1(k=1, \cdots, t)$ we obtain

$$
\sum_{j=1}^{m} \sum_{i=1}^{T}\left|\theta_{i j}\right|^{2}=\sum_{J} \sum_{k=1}^{t} \sum_{\lambda=1}^{n}\left|z_{k \lambda} \theta_{k J}\right|^{2}=\sum_{J} \sum_{k=1}^{t}\left|\theta_{k J}\right|^{2} .
$$

The relation (4.5) with the aid of (4.6), (4.7) and

$$
P\left(\sum_{j=1}^{m}\left|F_{j}\right|^{2}\right) \leq \sum_{j_{1}<\cdots<j_{n}} P\left(\sum_{\lambda=1}^{n}\left|F_{j_{\lambda}}\right|^{2}\right)
$$

implies (4.2) and (4.3).

4.3. We are now in the position to prove Theorem 2. We may assume that $m \geq n$. If not, then we set $f_{m+1}=\cdots=f_{n}=f_{m}$. For $F_{j}=f_{j}(j=1$, $\cdots, m)$ we take $\theta_{i j}$ in $H^{\infty}(\bar{D})$ and $\tau_{i}$ in $H^{\infty}(\bar{R})(i=1, \cdots, T ; j=1, \cdots, m)$ determined in Lemma 8 . Since

$$
P\left(\sum_{j=1}^{m}\left|f_{j}\right|^{2}\right)^{2} \geq P\left(|g|^{2}\right)=|P(g)|^{2},
$$

if we set

$$
\varphi=\sum_{i=1}^{T} \sum_{j=1}^{m}\left|\theta_{i j}\right|^{2} \quad \text { and } \quad \psi_{i j}=\overline{\theta_{i j}} \frac{P(g)}{\varphi}
$$

then

$$
\varphi=\sum_{i=1}^{T} \sum_{j=1}^{m}\left|\theta_{i j}\right|^{2} \geq C_{0}|P(g)|^{2}, \quad \sum_{i=1}^{T} \sum_{j=1}^{m} \psi_{i j} \tau_{i} f_{j}=P(g)
$$

and

$\psi_{i j}$ is smooth on $\bar{D}$ and $\left|\psi_{i j}\right| \leq C_{0}^{-1 / 2}$.

By the proof of Theorem 2.3 of [Ga, p. 329], there exist functions $b_{i j, \alpha \beta}$ $(i, \alpha=1, \cdots, T ; j, \beta=1, \cdots, m)$ continuous on $\bar{D}$ and smooth on $D$ such that

$$
\frac{\partial}{\partial \bar{z}} b_{i j, \alpha \beta}=P(g) \psi_{i j} \frac{\partial}{\partial \bar{z}} \psi_{\alpha \beta}
$$

and

$$
\left\|b_{i j, \alpha \beta}\right\|=\sup \left\{\left|b_{i j, \alpha \beta}(z)\right|:|z|=1\right\} \leq C
$$

where $C$ is some constant depending on $m$ and $n$. If we set

$$
h_{i j}=P(g)^{2} \psi_{i j}+\sum_{\alpha=1}^{T} \sum_{\beta=1}^{m}\left(b_{i j, \alpha \beta}-b_{\alpha \beta, i j}\right) \tau_{\alpha} f_{\beta},
$$


then

$$
\sum_{i=1}^{T} \sum_{j=1}^{m} h_{i j} \tau_{i} f_{j}=P(g)^{3}
$$

and

$$
\frac{\partial}{\partial \bar{z}} h_{i j}=0
$$

except for branch points. Since any $h_{i j}$ is smooth all over $R, h_{i j}$ is holomorphic on $R$. The functions

$$
h_{j}=\sum_{i=1}^{T} h_{i j} \tau_{i} \quad(j=1, \cdots, m)
$$

are the required.

\section{§5. Proof of Theorem 3}

5.1. In this section we prove Theorem 3 . The function $g$ satisfies the following equation:

$$
g^{n}+a_{1} g^{n-1}+\cdots+a_{n-1} g+a_{n}=0,
$$

where each $a_{k}$ belongs to $H^{\infty}(D)$. Let

$$
\Phi(X)=X^{n}+a_{1}(z) X^{n-1}+\cdots+a_{n-1}(z) X+a_{n}(z) .
$$

If $\pi^{-1}(z)=\left\{p, p_{1}, \cdots, p_{n-1}\right\}$, then we have

$$
\Phi(X)=(X-g(p))\left(X-g\left(p_{1}\right)\right) \cdots\left(X-g\left(p_{n-1}\right)\right) .
$$

Therefore we have

$$
\frac{d^{k}}{d X^{k}} \Phi(g(p))=C(n, k) \sum_{i_{1}<\cdots<i_{n-k}} \sum_{\lambda=1}^{n-k}\left(g(p)-g\left(p_{i_{2}}\right)\right)
$$

where $C(n, k)$ is some constant depending only on $n$ and $k$. Since any zero of the Blaschke product $B(z)$ is simple, $B(z)$ is univalent about each zero point. Since the surface $R$ is defined by $\zeta=B(z)^{1 / n}$, for any branch point $p_{0}$ there exist a chart $\{U, \varphi\}$ such that $\varphi(U)=\{|w|<1\}, B^{1 / n} \circ \varphi^{-1}(w)$ $=w, \varphi\left(p_{0}\right)=0$ and $\varphi(p)^{n}=\varphi\left(p_{i}\right)^{n}(i=1, \cdots, n-1)$ if $\pi^{-1}(z) \subset U$. A function $g \circ \varphi^{-1}(w)$ is bounded holomorphic on $\{|w|<1\}$. By the Schwarz lemma, there exists a constant $C_{1}$ such that

$$
\left|g \circ \varphi^{-1}(w)-g \circ \varphi^{-1}(0)\right|<C_{1}|w| .
$$


Hence we have, for $p$ and $p_{i}$ on $\pi^{-1}(z) \subset U$,

$$
\begin{aligned}
\left|g(p)-g\left(p_{i}\right)\right| & \leq\left|g \circ \varphi^{-1}(\varphi(p))-g \circ \varphi^{-1}(0)\right|+\left|g \circ \varphi^{-1}\left(\varphi\left(p_{i}\right)\right)-g \circ \varphi^{-1}(0)\right| \\
& \leq C_{1}|\varphi(p)|+C_{1}\left|\varphi\left(p_{i}\right)\right| \leq 2 C_{1}|\varphi(p)|=2 C_{1}\left|B^{1 / n}(p)\right| .
\end{aligned}
$$

Since functions of (5.3) belong to $H^{\infty}(R)$, there exist $s_{1}, \cdots, s_{n-1}$ in $H^{\infty}(R)$ such that

$$
\frac{d^{k}}{d X^{k}} \Phi(g)=s_{k} B^{(n-k) / n} \quad(k=1, \cdots, n-1)
$$

Hence we have

$$
\frac{n !}{(n-k) !} g^{n}+\sum_{k=1}^{n-k} \frac{(n-j) !}{(n-k-j) !} g^{n-j} a_{j}=s_{k} g^{k} B^{(n-k) / n} .
$$

By the maximum principle, each $s_{k}$ satisfies (2.6). By induction we will prove the following equations:

$$
\begin{aligned}
& \frac{(n-1) !}{(n-k) !} g^{n}+\sum_{j=1}^{n-k} \frac{(n-1-j) !}{(n-k-j) !} g^{n-j} a_{j} \\
& \quad=\sum_{j=1}^{k-1} s_{n-j, k} g^{j} B^{(n-j) / n}+(-1)^{k}(k-1) ! a_{n},
\end{aligned}
$$

where $s_{n-j, k}$ are elements of $H^{\infty}(R)$. When $k=1$, by (5.1) we see that $(5.5: 1)$ holds. Suppose that $(5.5: k)$ holds. By $(5.4: k)$ and $(5.5: k)$, we have $(5.5: k+1)$. Consequently we have

$(5.5: n) \quad(n-1) ! g^{n}=\sum_{j-1}^{n-1} s_{n-j, n-1} g^{j} B^{(n-j) / n}+(-1)^{n}(n-1) ! a_{n}$.

Since $a_{n}=(-1)^{n} P(g)$, Theorem 3 follows.

\section{§6. Proof of Theorem 4}

6.1. For the proof of Theorem 4 we need the following key lemma:

LEMma 9. There exists a positive integer valued function $m=m(a)$ defined on the open interval $(0,1)$ such that

$$
\left|\frac{z-a}{1-a z}\right|^{m} \leq 2\left\{|1-| z+\left|\frac{z-a}{1-a z}\right|^{n+m}\right\}
$$

on $D$ and that

$$
\lim _{a \rightarrow 1} a^{m}=1
$$


Proof. Let

$$
K_{a}=\left\{2|(z-a) /(1-a z)|^{n} \leq 1\right\} .
$$

On $D \backslash K_{a}$ the inequality appearing in (6.1) holds for any positive integer $m$. Take a number $c$ such that $a<c<1$ and $(c-a) /(1-a c)=2^{-1 / n}$. We have

$$
1-a=\frac{1+a 2^{-1 / n}}{1-2^{-1 / n}}(1-c) \leq 2\left(1-2^{-1 / n}\right)^{-1}(1-c) .
$$

Since

$$
|(z-a) /(1-a z)|^{n \lambda} \leq 2^{-\lambda}
$$

on $K_{a}$, if we select $\lambda=\lambda(a)$ such that

$$
2^{-\lambda} \leq(1 / 2)\left(1-2^{-1 / n}\right)(1-a)
$$

and

$$
\lim _{a \rightarrow 1} a^{n \lambda}=1
$$

then we have, on $K_{a}$,

$$
\left|\frac{z-a}{1-a z}\right|^{n \lambda} \leq(1 / 2)\left(1-2^{-1 / n}\right)(1-a) \leq 1-c \leq|1-z| .
$$

We set

$$
\lambda=1+\text { integral part of }-\left(C_{1} \log (1-a)+C_{2}\right)
$$

where $C_{1}=(\log 2)^{-1}$ and $C_{2}=(\log 2)^{-1} \log \left(2^{-1}\left(1-2^{-1 / n}\right)\right)$. Since

$$
\lim _{a \rightarrow 1}(\log (1-a))(\log a)=0,
$$

the function $\lambda=\lambda(a)$ satisfies (6.4) and (6.5) so that $m=n \lambda(a)$ satisfies (6.1) and (6.2).

6.2. We now prove Theorem 4. Since $K_{a}$ of (6.3) is a compact subset of $D$, for any $a, 0<a<1$, by Lemma 9 , there exist finite Blaschke products $B_{a}$ and $G_{a}$ satisfying the following three properties:

(1) $G_{a}$ has a single simple zero at $a$;

(2) any zero of $B_{a}$ is simple and contained in a sufficiently small neighborhood of $a$;

(3) $B_{a}$ and $G_{a}$ satisfy 


$$
\left|B_{a}(z)\right| \leq 3\left\{|1-z|+\left|B_{a}(z) G_{a}(z)^{n}\right|\right\}
$$

on $D$ and

$$
\lim _{a \rightarrow 1} B_{a}(0)=\lim _{a \rightarrow 1} G_{a}(0)=1 .
$$

By induction we will select a positive increasing sequence $\left\{a_{N}\right\}$ converging to 1 such that if we set

$$
B_{N}=\prod_{k=1}^{N} B_{a_{k}} \quad \text { and } \quad G_{N}=\prod_{k=1}^{N} G_{a_{k}}
$$

then $B_{N}$ and $G_{N}$ satisfy the following two properties:

( $\alpha$ ) Any zero of $B_{N}$ is simple;

( $\beta$ ) $B_{N}$ and $G_{N}$ satisfy

$$
\left|B_{N}(z)\right| \leq(4-1 / N)\left\{|1-z|+\left|B_{N}(z) G_{N}(z)^{n}\right|\right\}
$$

on $D$ and

$$
\left|B_{N}(0)\right|>1 / 2, \quad\left|G_{N}(0)\right|>1 / 2 .
$$

We choose an arbitrary $a_{1}$ in $(0,1)$ such that $\left|B_{a_{1}}(0)\right|,\left|G_{n_{1}}(0)\right|>1 / 2$. This determines $B_{1}$ and $G_{1}$. Suppose that $G_{N-1}$ and $B_{N-1}$ are constructed. We set $c=3(4-1 / N)^{-1}$. Let

$$
K=\left\{\left|B_{N-1}(z) G_{N-1}(z)^{n}\right| \leq c\right\} .
$$

Since $K \cup\{0\}$ is a compact subset of $D$, by (6.8), if $a$ is sufficiently close to 1 , then $B_{a}(z) G_{a}(z)$ is close to 1 uniformly on $K \cup\{0\}$. Since $\max (|1-z|$; $z \in K\}>0$, we can select $a$ in $(0,1)$ such that if we set

$$
B_{N}(z)=B_{N-1}(z) B_{a}(z) \text { and } G_{N}(z)=G_{N-1}(z) G_{a}(z),
$$

then the inequality (6.10) holds on $K$ and (6.11) and $(\alpha)$ are satisfied. For any $z$ in $D \backslash K$ we have

$$
\begin{aligned}
\left|B_{N}(z)\right| & =\left|B_{N-1}(z) B_{a}(z)\right| \leq\left|B_{a}(z)\right| \leq 3\left\{|1-z|+\left|B_{a}(z) G_{a}(z)^{n}\right|\right\} \\
& \leq(4-1 / N)\left\{1-z|+| B_{N}(z) G_{N}(z)^{n} \mid\right\},
\end{aligned}
$$

since $\left|B_{N}(z) G_{N}(z)^{n}\right| \geq c\left|B_{a}(z) G_{a}(z)^{n}\right|$. If we set

$$
B(z)=\lim _{N \rightarrow \infty} B_{N}(z) \text { and } G(z)=\lim _{N \rightarrow \infty} G_{N}(z),
$$

then $B$ and $G$ are exactly what we required. 


\section{Appendix}

A.1. We will prove (1.5). Suppose $w(R)<\infty$, otherwise the assertion is trivial. Take an arbitrary $f_{1}, \cdots, f_{m}$ and $g$ in $H^{\infty}(D)$ with (1.1). Viewing these as in $H^{\infty}(R)$ there exist $m$ functions $h_{1}, \cdots, h_{m}$ in $H^{\infty}(R)$ with

$$
\sum_{j=1}^{m} f_{j} h_{j}=g^{w(R)}
$$

on $R$. By taking the means of the above for $n$ points $p_{k}$ in $\pi^{-1}(z)$ we obtain

$$
\sum_{j=1}^{m} f_{j} A\left(h_{j}\right)=g^{w(R)}
$$

on $D$ where $A\left(h_{1}\right), \cdots, A\left(h_{m}\right)$ belong to $H^{\infty}(D)$. Hence we deduce (1.5).

A.2. We will prove (1.6). Take an arbitrary $f_{1}, \cdots, f_{m}$ and $g$ in $H^{\circ}(R)$ with (1.1). Let $(D, R, \tau)$ be the universal covering surface of $R$. Then $f_{1} \circ \tau, \cdots, f_{m} \circ \tau$ and $g \circ \tau$ belong to $H^{\infty}(D)$ with (1.1). Hence there exist $m$ functions $h_{1}, \cdots, h_{m}$ in $H^{\infty}(D)$ with

$$
\sum_{j=1}^{m}\left(f_{j} \circ \tau\right) h_{j}=(g \circ \tau)^{w(D)}
$$

on $D$. Take the Forelli projection $\mathscr{F}: H^{\infty}(D) \rightarrow H^{\infty}(R)$ (see [Fo], [F]) of the both sides of the above using the additivity and the $H^{\infty}(R)$-homogeneity of $\mathscr{F}$. Then we obtain

$$
\sum_{j=1}^{m} f_{j} \mathscr{F}\left(h_{j}\right)=g^{w(D)}
$$

on $R$ where $\mathscr{F}\left(h_{1}\right), \cdots, \mathscr{F}\left(h_{m}\right)$ belong to $H^{\infty}(R)$. Hence we see that $w(R)$ $\leq w(D)$. This with (1.5) implies (1.6).

\section{REFERENCES}

[A1] Alling, N., A proof of the corona conjecture for finite open Riemann surfaces, Bull. Amer. Math. Soc., 70 (1964), 110-112.

[A2] - Extensions of meromorphic function rings over non-compact Riemann surfaces. I, Math. Z., 89 (1965), 273-299.

[B1] Behrens, M., The corona conjecture for a class of infinitely connected domains, Bull. Amer. Math. Soc., 76 (1970), 387-391.

[B2] - The maximal ideal space of algebras of bounded analytic functions on infinitely connected domains, Trans. Amer. Math. Soc., 161 (1971), 359-379.

[BR] Berndtsson, B. and T. Ransford, Analytic multifunctions, the $\bar{\partial}$-equation and a proof of the corona theorem, Pacific J. Math., 124 (1986), 57-72. 
[C1] Carleson, L., Interpolations by bounded analytic functions and the corona problem, Ann. of Math., 76 (1962), 547-559.

[C2] - On $H^{\infty}$ in multiply connected domains, Conference on harmonic analysis in honor of Antoni Zygmund, Vol II, Beckner W., et al., Wadsworth, 1983, 349372.

[Ce] Cegrell, U., A generalization of the corona theorem in the unit disc, Math. Z., 203 (1990), 255-261.

[D] Deeb, W., A class of infinitely connected domains and the corona, Trans. Amer. Math. Soc., 231 (1977), 101-106.

[DW] Deeb, W. and D. Wilken, s-domains and the corona, Trans. Amer. Math. Soc., 231 (1977), 107-115.

[EM1] Earle, C. and A. Marden, Projections to automorphic functions, Proc. Amer. Math. Soc., 19 (1968), 274-278.

[EM2] - - On Poincaré series with application to $H^{p}$ spaces on bordered Riemann surfaces, Illinois J. Math., 13 (1969), 202-219.

[F] Fisher, S., Function Theory on Planar Domains, Wiley-Interscience, 1983.

[Fo] Forelli, F., Bounded holomorphic functions and projections, Illinois J. Math., 10 (1966), 367-380.

[G1] Gamelin, T., Localization of the corona problem, Pacific J. Math., 34 (1970), 73-81.

[G2] —- Uniform Algebras and Jensen Measures, London Math. Soc. Lecture Note Series, 32, 1978.

[G3] - - Wolff's proof of the corona problem, Israel J. Math., 37 (1980), 113-119.

[Ga] Garnett, J., Bounded Analytic Functions, Academic Press, 1981.

[GJ] Garnett, J. and P. Jones, The corona theorem for Denjoy domains, Acta Math., 155 (1985), 27-40.

[GM] Gorkin, P. and R. Mortini, F-ideals in $Q A_{B}$ J. London Math. Soc., (2) 37 (1988), 509-519.

[H1] Hara, M., The corona problem on 2-sheeted disks, Proc. Japan Acad., 58 (1982), 256-257.

[H2] - - On Camelin constants, Pacific J. Math., 110 (1984), 77-81.

[HN] Hara, M. and M. Nakai, Corona theorem with bounds for finitely sheeted disks, Tôhoku Math. J., 37 (1985), 225-240.

[Ha] Hatori, O., On T. Wolff's problem (in Japanese), The Scientific Researches Math. (The School of Education, Waseda Univ.), 33 (1984), 41-49.

[JM] Jones, P. and D. Marshall, Critical points of Green's function, harmonic measures, and the corona problem, Ark. Math., 23 (1985), 281-314.

[K] Koosis, P., Introduction to $H^{p}$ Spaces, London Math. Soc., Lecture Note Series 40, 1980.

[M] Moore, C., The corona theorem for domains whose boundary lies in a smooth curve, Proc. Amer. Math. Soc., 100 (1987), 266-270.

[Mo] Mortini, R., Corona theorems for subalgebras of $H^{\infty}$ Michigan Math. J., 36 (1989), 193-202.

[N1] Nakai, M., Corona problem for Riemann surfaces of Parreau-Widom type, Pacific J. Math., 103 (1982), 103-109.

[N2] - The corona problem on finitely sheeted covering surfaces, Nagoya Math. J., 92 (1983), 163-173.

[Na] Narita, J., A remark on the corona problem for plane domains, J. Math. Kyoto Univ., 25 (1985), 293-298.

[R] Rao, K., On a generalized corona problem, J. Analyse Math., 18 (1967), 277-278.

[SN] Sario, L. and M. Nakai, Classification Theory of Riemann Surfaces, Berlin- 
Heidelberg-New York: Springer, 1970.

[S1] Slodkowski, Z., An analytic set-valued selection and its applications to the corona theorem, to polynomical hulls and joint spectra, Trans. Amer. Math. Soc., 294 (1986), 367-377.

[S2] - - On bounded analytic functions in finitely connected domains, Trans. Amer. Math. Soc., 300 (1987), 721-736.

[St1] Stout, E., Two theorems concerning functions holomorphic on multiply connected domains, Bull. Amer. Math. Soc., 69 (1963), 527-530.

[St2] - - Some theorems on bounded holomorphic functions, Bull. Amer. Math. Soc., 70 (1964), 419-421.

[St3] —- Bounded holomorphic functions on finite Riemann surfaces, Trans. Amer. Math. Soc., 120 (1965), 255-285.

[Z] Zalcman, L., Bounded analytic functions on domains of infinite connectivity, Trans. Amer. Math. Soc., 144 (1969), 241-269.

Department of Mathematics

Meijo University

Shiogamaguchi, Tenpaku, Nagoya 468

Japan 